\title{
REHABILITATION OF REPAIRED FLEXOR TENDONS OF THE HAND: THERAPISTS' PERSPECTIVE
}

\begin{abstract}
INTRODUCTION: Successful outcomes following flexor tendon repairs in the hand are dependent on a team approach to management and rehabilitation. In South Africa, the public sector therapists' perspective on rehabilitation of the hand has not been explored.
\end{abstract}

PURPOSE: This study determined the public sector therapists' perspective on rehabilitation of patients with flexor tendon repairs in two districts of one province in South Africa. Practices in urban and rural settings were compared METHODS: A cross sectional survey accessed urban and rural public sector therapists involved in the rehabilitation of repaired flexor tendons of the hand. The questionnaire sought information on the initiation and frequency of rehabilitation, initiation of active movement exercises, initiation of resistive exercises, discontinuation of protective splints, the use and presence of flexor tendon protocols in the facility, proximal interphalangeal joint (PIP) joint contractures, suture technique, work experience and choice of protocol.

RESULTS: Therapists worked closely with physicians and found comfort in the availability of protocols. The majority of therapists in regional (93\%) and tertiary hospital (94\%) used established protocols compared to their district hospital counterparts (p<0, 05). External attributions were more significant in making key decisions for rehabilitation. The Strickland formula was not used as an outcome measure.

CONCLUSION: Therapists are not confident in managing repaired flexor tendons of the hand. Therapists in urban health facilities rely on established rehabilitation protocols compared to their rural counterparts. Team work is evident in both settings.

KEYWORDS: REHABILITATION PRACTICES, HAND THERAPY, FLEXOR TENDON REPAIR

\section{INTRODUCTION}

Flexor tendon injuries are a common, but complex problem that can lead to significant functional disability despite being managed timeously and appropriately (Jones and Laing 2006). Successful outcomes are dependent not only on a skillful surgical technique, but also on the subsequent precise rehabilitation and compliance of the patient (Groth 2008, Tidsskr 2008). Despite considerable research and clinical efforts, the successful management of flexor tendon repairs remains elusive (Tang 2007, Torie et al 2010). Satisfactory and unpredictable

\section{CORRESPONDING AUTHOR:}

Puckree T

Faculty of Health Sciences,

Gate 8, Steve Biko Road,

Durban University of Technology,

Durban

E-mail: puckreet@dut.ac.za functional outcomes continue to impact on patients' activities and participation (Oltman et al 2008, Tang 2007). The surgical technique has to be complimented by a precise therapeutic approach (Groth 2005). Lilly and Messer (2006) suggested that meticulous surgical technique and early postoperative tendon mobilization in a well-supervised therapy program can minimize the frequency and severity of complications such as adhesions.

Therapists feel daunted, confused, and apprehensive about managing a hand following primary repair of a flexor tendon (Braga-Silva and Kuyven 2005). Groth (2008) confirms that the availability of protocols eases feelings of apprehension, promoting a more precise approach to rehabilitation. Therapists work with surgeons to choose the postoperative tendon management protocol (Mackin et al 2002). Groth (2008) suggests the use of established protocols such as the Modified Kleinert or Duran to make clinical decisions, thereby simplifying the task of clinicians who manage flexor tendons postoperatively. Edinburg et al (1987) reported that there are different practices or modifications of the existing protocols in different hospital settings. Protocols can be modified according to the environment that patients come from (Mackin et al 2002).

In South Africa, the scarcity of occupational and physiotherapists poses a challenge to rehabilitation in general (Mars 2011) and to hand rehabilitation specifically. All public sector therapists are expected to manage any patient, regardless of experience or training. To ensure that patients with repaired flexor tendons receive at least a minimal standard of care it is important to determine the status of hand rehabilitation in public facilities from the perspective of the therapist. Groth (2005), in a survey of flexor tendon rehabilitation relating to practice patterns, attitudes and autonomy 
in Canada, reported that therapists allocate $30 \%$ of their time to rehabilitation of flexor tendon disorders, and have identified this area as a research priority, which may not be the case in South Africa.

This study seeks to determine the public sector therapists' perspective on rehabilitation of patients with flexor tendon repairs in two districts of one province in South Africa.

\section{METHODS}

A cross sectional survey was conducted on physiotherapists and occupational therapists working in public hospitals in one rural and one urban district in one of the nine provinces in South Africa. Districts were chosen by convenience to the investigator. Through the use of convenience sampling, only hospitals that received patients requiring flexor tendon care and all therapists managing these patients were invited to participate in the study. The rural district has only one regional hospital that performs flexor tendon surgery, and in the urban district five of the participating hospitals offer this service. A questionnaire, modified from the one used by Groth (2005), included closed-ended questions relating to the initiation and frequency of rehabilitation, initiation of active movement exercises, initiation of resistive exercises, discontinuation of protective splints, the use and presence of flexor tendon protocols in the facility, proximal interphalangeal joint (PIP) joint contractures, suture technique, work experience and choice of protocol. Respondents were requested to choose more than one option provided for each question if required. The questionnaire was validated using an expert group and a pilot study. All questionnaires were administered by the one investigator, to ensure reliability of the data. All gatekeeper permissions and fully informed consents supplemented approval by the Institutional Research Ethics Committee to conduct the study. Descriptive statistics such as means, and standard deviations were computed using SPSS version 19. Pearson Chi-square test was used to test for association between practices and districts. Independent samples t-tests were used to test for differences in continuous variables between districts. The probability was set at $\mathrm{p}<0,05$.

\section{RESULTS}

A $100 \%$ response rate was obtained through a return of 63 completed questionnaires. Of these $26.9 \%$ therapists worked in rural district hospitals and $73.1 \%$ in the urban district. A total of $22 \%$ of the participants (five of the therapists from the rural and nine from the urban district) indicated that they had not seen patients with flexor tendon repairs in the past five years.

The respondents were primarily physiotherapists $(75.8 \%)$ with the remainder being occupational therapists. Participants worked in a variety of hospital environments namely tertiary (26.5\%), regional $(57.1 \%)$, and district hospitals (14.3\%) and clinics $(2.1 \%)$. Significantly more therapists attended to adult outpatients (91. 9\%; p < 0.05).

The rehabilitation protocols chosen were based on the needs of individual patients. As shown in Table 1, therapists did not adhere to the use of a single protocol. Established protocols were used more commonly by therapists in tertiary and regional hospitals in the urban district compared to therapists in rural district hospitals who treated patients symptomatically. Regional hospitals favoured the use of the immobilization protocol. A significant difference between tertiary, regional and district hospitals in the use of the Duran protocol $(\mathrm{p}=0.003)$ and immobilization $(\mathrm{p}=0.045)$ protocols was noted. The majority of the therapists from the rural district indicated that they do not have flexor tendon protocols/ clinical guidelines in their departments.

\section{Initiation of therapy}

As shown in Table 2, the decision to initiate therapy was never taken solely by the therapist but either by the doctor $(31.3 \%)$ or collaboratively $(68.8 \%)$ by the doctor and therapist. Only the initiation of resistance was decided by the therapist independently, significantly more frequently. There was no statistical difference $(p=0.196)$ on the decision to initiate therapy amongst different hospital settings.

The majority of respondents $(83 \%)$ reported that they initiated rehabilitation between day one and day three after the repair with minimal difference in practice between levels of hospitals. More of the respondents $(70.2 \%)$ initiated therapy only on orders from the doctor. More than two thirds of the respondents $(68.8 \%)$ reported that the doctor and the therapist decided on when therapy should begin (Table 2) compared to $44.7 \%$ who initiated therapy using established protocols. Therapy was initiated from day one to three by $83 \%$ of therapists working in the urban district and $75.6 \%$ of therapists from the rural district.

\section{Frequency of therapy visits}

The majority of the respondents $(85 \%)$ reported that the therapist decides on the frequency of therapy visits (Table $2)$. Although doctors were involved in deciding on the frequency of therapy visits, therapists made this decision significantly more often. Patients were treated once per week $(37.5 \%)$, and twice a week (25\%). Approximately $40 \%$ believed that patients' appointments should be scheduled twice a week in the first month. Frequency of therapy visits did not differ by hospital setting but was significantly related to the incidence of PIP joint contractures $(p=0.013)$. Some therapists reported seeing patients with PIPJ contractures even though therapy visits occurred once and twice a week. Therapists from the rural district (58.3\%) scheduled therapy visits once a week compared to $29.7 \%$ of the urban therapists.

\section{Initiation of active range of movement}

Active range of movement (ROM) exercises was initiated from the fourth post-operative week $(29.2 \%)$, 1st and 3 rd week $(20.8 \%)$, 5th week $(12.5 \%)$ and second week (16.7\%). A significant difference $(p=0.002)$ in the initiation of active range of movement by hospital setting was noted with active movement initiated between day seven and thirteen by about $50 \%$ of the therapists from tertiary institutions, day zero and six by $80 \%$ of therapists from regional hospitals and 14-20 days by $40 \%$ of therapists from the district hospitals. A large number of rural therapists $(41.6 \%)$ reported initiating active ROM between day zero and day six post operatively compared to the $13.5 \%$ of the urban district therapists. As shown in Table 3, external attributions such as established protocol, and number of postoperative days was cited by the majority of the respondents as important for initiating active ROM, initiating resistance and discontinuing the orthoses.

\section{Discontinuation of protective splinting}

Custom protective orthoses were commonly fabricated by the occupational therapists. The wrist was positioned at 20 to 30 degrees of flexion by $31.8 \%$ of the 


\begin{tabular}{|l|l|l|l|l|l|}
\hline \multirow{2}{*}{$\begin{array}{l}\text { Flexor Tendon } \\
\text { Protocol }\end{array}$} & \multicolumn{3}{|c|}{$\begin{array}{c}\text { Therapists(\%) in different hospital settings** } \\
\mathrm{nn} \\
\text { nn responses in \% }\end{array}$} & \multirow{2}{*}{ Total \% } & \multirow{2}{*}{ P Values } \\
\cline { 2 - 5 } & Tertiary & Regional & District & & \multirow{2}{*}{0.387} \\
\hline Kleinert Protocol & 26.7 & 63.3 & 10.0 & 61.2 & \multirow{2}{*}{$0.003^{*}$} \\
\hline Duran Protocol & 53.6 & 40.0 & 0 & 55.0 & \multirow{2}{*}{$0.045^{*}$} \\
\hline Immobilization & 18.5 & 74.1 & 7.2 & 30.6 & 0.754 \\
\hline Treat symptoms & 30 & 50 & 20 & 20.4 & \\
\hline Total & $13(100)$ & $28(100)$ & $7(100)$ & $49(100)$ & \\
\hline
\end{tabular}

** Therapist could indicate more than one protocol

*Indicates a significance difference between different hospital settings

respondents. The metacarpophalangeal joint (MPJ) was placed at 60-70 degrees by $24 \%$ of the respondents and $42 \%$ of the physiotherapists supplemented mobilization with ultrasound therapy and transcutaneous nerve stimulation (TENS).

About $37 \%$ of the respondents discontinued protective orthoses at five weeks and $31 \%$ at six weeks. A large number of respondents $(56.5 \%)$ felt that the therapist should decide when to discontinue protective splinting (Table 2). Some therapists $(22 \%)$ thought that protective splinting should be typically discontinued at four, five and six weeks postoperatively depending on the patient, the nature of injury and of the tendon repair. This opinion was significantly different by hospital setting ( $\mathrm{p}=0.004$ ): $80 \%$ of the therapists from regional hospitals discontinue splinting at six weeks, 55\% of tertiary hospital therapist discontinue splinting at five weeks, compared to 33.3 $\%$ of therapists at district hospitals who discontinue splinting at eight weeks.
Most of the therapists from the rural district (66.6) discontinued protective splinting on the 8th week post operatively compared to the urban district therapists $(51.3 \%)$ who discontinued splinting at six weeks postoperatively.

\section{Initiation of resistance exercises}

Opinions on when resistance exercises should begin included $18.4 \%$ at four weeks, $30.6 \%$ at five weeks, $22.4 \%$ at six weeks, $26.6 \%$ at seven weeks, and $2.0 \%$ indicated eight weeks. Timing of initiation of therapy was significantly different by hospital setting $(\mathrm{p}=0.010)$. Rural therapists $(41.1 \%)$ initiate resistance from the 8 th week and $24.6 \%$ of the urban therapists initiate resistance from the 6 th week post operatively.

\section{PIPJ Contracture}

The majority of the therapists $(52.1 \%)$ managed PIPJ contractures sometimes compared to $8.3 \%$ who treated these contractures nearly always. About a third $(31.3 \%)$ of the therapists seldom saw patients with PIPJ contracture.

\section{Outcomes}

All therapists measured at least one or more outcome measures with functional independence most commonly measured, compared to grip and pinch strength. None of the respondents used the Strickland's formula. More than half of the respondents $(56.8 \%)$ reported that they had never encountered a patient who suffered a postoperative tendon rupture.

\section{Attitudes}

Nearly half $(49 \%)$ of the therapists reported that they are sometimes apprehensive about how to progress patients through rehabilitation. Factors which contributed towards poor and unsuccessful patient outcomes included, non-compliance with home program $(83.7 \%)$, injury severity $(55.1 \%)$, and poor attendance at therapy sessions $(40.8 \%)$. Patients with successful out-

Table 2: Health care professionals $(\mathrm{N}=49)$ who decide on the key elements regarding therapy

\begin{tabular}{|l|l|l|l|c|}
\hline Key element & $\begin{array}{l}\text { Doctor } \\
\%\end{array}$ & $\begin{array}{l}\text { Therapist } \\
\%\end{array}$ & $\begin{array}{l}\text { Doctor and } \\
\text { therapist \% }\end{array}$ & P Values \\
\hline Initiation of therapy & $31.3 \%$ & $0 \%$ & $68.8 \%$ & 0.196 \\
\hline Frequency of therapy visits & $2.1 \%$ & $85.1 \%$ & $42.9 \%$ & 0.438 \\
\hline Initiation of active movement & $12.2 \%$ & $44.9 \%$ & $42.9 \%$ & 0.568 \\
\hline Discontinuation of splinting & $0 \%$ & $56.5 \%$ & $43.5 \%$ & $0.010 *$ \\
\hline Initiation of resistance & $2.2 \%$ & $69.6 \%$ & $28.3 \%$ & \\
\hline $\begin{array}{l}* \text { Indicates significance } p<0.05 \\
* * \text { Therapist could indicate more than one protocol }\end{array}$ & & & \\
\hline
\end{tabular}


Table 3: Responses $\mathrm{N}=49$ (\% of participants) regarding external and internal attribution factors informing decisions on the use of specific protocols

\begin{tabular}{|l|l|l|l|l|}
\hline \multirow{2}{*}{ Attribution } & \multirow{2}{*}{ Factors } & \multicolumn{2}{|l|}{ Three key elements: therapists responses in \% } \\
\cline { 3 - 5 } & & Initiation of Active ROM & $\begin{array}{l}\text { Discontinuation of } \\
\text { protective Splint }\end{array}$ & Initiation of Resistance \\
\hline \multirow{3}{*}{$\begin{array}{l}\text { External } \\
\text { attribution }\end{array}$} & Established protocol & $61.2 \%$ & $56.3 \%$ & $66.7 \%$ \\
\cline { 2 - 5 } & No. of postoperative days & $38.8 \%$ & $47.9 \%$ & $45.8 \%$ \\
\cline { 2 - 5 } & Doctor's order & $32.7 \%$ & $20.8 \%$ & $14.6 \%$ \\
\hline \multirow{3}{*}{$\begin{array}{l}\text { Internal } \\
\text { attribution }\end{array}$} & Suture technique & $12.2 \%$ & $8.3 \%$ & $12.5 \%$ \\
\cline { 2 - 5 } & Compliance issues & $16.3 \%$ & $20.8 \%$ & $10.4 \%$ \\
\cline { 2 - 5 } & ROM measurement & $8.2 \%$ & $18.8 \%$ & $14.6 \%$ \\
\cline { 2 - 5 } & Other & $4.1 \%$ & $2.2 \%$ & $4.2 \%$ \\
\hline \multirow{2}{*}{$\begin{array}{l}\text { Participants were instructed to tick all applicable choices } \\
\text { ROM= range of movement }\end{array}$} & & & \\
\hline
\end{tabular}

comes were compliant with: the home exercise program $(89.8 \%)$, with splinting $(42.9 \%)$ and individualized rehabilitation $(38.8 \%)$. A significant number $(86.6 \%)$ of therapists reported that the place of patient's employment affected the frequency of therapy visits.

\section{Discussion}

This study revealed that the majority of patients with flexor tendon repairs were seen by therapists who work at regional hospitals. This finding could be related to the structure of the health care system in this province. The regional hospitals cater for larger numbers of patients with more complicated conditions.

The participants in this study consisted of more physiotherapists than occupational therapists, which is different from the Groth (2005) study. Apart

from the fact that the public health sector employs more physiotherapists, Groth (2005) used a convenient sample of hand therapists compared to the saturation sample in this study.

Although the number of hospitals invited to participate in the study in the two districts was similar, in the urban district more of the participating hospitals (five) provided flexor tendon surgery compared to the rural district which had only one regional hospital that performs these operations.

The choice of rehabilitation protocols was dependent on the availability of the protocols, experience of the therapist and the actual needs of the patient. The fact that ten of the participants have less than one year of experience suggests that these therapists were serving their one year of community service and were

Table 4: Numbers and percentages of therapists who measured different outcomes

\begin{tabular}{|l|l|}
\hline Outcome measured & Number (\%) of therapists \\
\hline Functional independence & $34(70.8)$ \\
\hline ROM & $30(62)$ \\
\hline Grip strength & $18(37.5)$ \\
\hline Pinch strength & $14(29.2)$ \\
\hline Stricklands formula & $0(0)$ \\
\hline Other & $6(12.5)$ \\
\hline Participants were instructed to tick all applicable choices \\
\hline
\end{tabular}

relatively inexperienced in the management of patients with tendon repairs. Six of these therapists work in hospitals in the rural district compared to the four from the urban district. The symptomatical management of patients in rural district hospitals could suggest a lack of experience or training or simply being daunted, as described by Torie et al (2010). The use of established protocols by the majority of therapists in tertiary and regional hospitals may suggest both experience and continuous exposure to new trends and continuous professional education as well as the compliance to minimal standards of care. The findings of this research concur with the literature (Groth 2005, Mackin et al 2002, Rosenthal and Stoddard 2005), in that therapists in different hospital settings use different protocols to treat patients with flexor tendon injuries postoperatively. Mackin et al (2002) suggest that every tendon management is unique and often the best approach may include a combination of techniques from various protocols. In this study therapists either treated patients symptomatically or by using a combination of protocols. In regional hospitals where the majority of flexor tendons were repaired on site, therapists used established protocols such as the immobilization or Kleinert protocols.

However the findings of this study are similar to the findings of Groth (2005), who also found that the Kleinert-type of exercises is used more than the other protocols. The literature still emphasizes that a 
definitive management protocol has yet to be established (Tang 2007, Torie et al 2010).

Modifications to the established protocols are common, in order for the protocol to suit a particular clinic or setting (Groth 2008, Groth 2005, Sueoka and LaStayo 2008, Tang 2007). Although the current study found that therapists used combinations of approaches, more research is needed to develop different approaches and modifications to the established protocols, to be relevant and suitable for the South African community.

This study showed that the majority of therapy decisions were taken by therapists even though in rural district hospitals instructions by the surgeon are followed. These findings concur with the findings by Groth (2008) who highlighted that there is an expectation that therapists will make autonomous clinical decisions regarding the optimal rehabilitation interventions. Protocols or clinical guidelines that are developed and approved by the surgeons can assist the therapists in these decisions even if the surgeon is not there during rehabilitation. Groth (2008) also suggested that the use of established protocols is the most commonly reported clinical reasoning strategy. Our study found that external attributions such as established protocols are cited as significant for the initiation of active movement, and also important in the decisions which concerned discontinuation of protective splinting and initiation of resistance. The initiation of therapy by urban and rural therapists in the first week (between day one and three) after the repair was similar to the findings of Groth (2005). Groth also reported that this practice fluctuated quite frequently. Therapists reported that they typically see their patients once $(37.5 \%)$ and twice $(35 \%)$ per week. In this study, therapists believed that therapy visits should be scheduled twice a week. Groth (2005) found that private hospital care settings such as 'physician-owned or hospital-owned' settings, were more likely to schedule patients' appointments twice a week. In public hospitals where this study was conducted, twice a week appointments may be too demanding both on patient and hospital resources. Patient compliance to therapy visits may become a problem with twice a week appointments.

Proximal interphalangeal joint con-

Table 5: Comparison of the initiation of the key elements in the two districts

\begin{tabular}{|l|l|l|}
\hline & \multicolumn{2}{|l|}{ Number (\%) participants } \\
\hline Key element & Rural district & Urban district \\
\hline Initiation of therapy: day 1-3 & $10(58.8)$ & $28(60.8)$ \\
\hline Frequency of therapy visits: 1x/week & $7(41.1)$ & $11(23)$ \\
\hline Initiation of active movement: day 0-6 & $5(29.4)$ & $5(10.9)$ \\
\hline Discontinuation of splinting: 6th week & $2(11.8)$ & $7(15.2)$ \\
\hline Initiation of resistance: 6 th week & $1(5.9)$ & $8(17.4)$ \\
\hline
\end{tabular}

Participants were instructed to tick all applicable choices

tractures were noted regardless of setting even though therapy visits occurred once and twice a week. These contractures were seen less often in patients who were seen once a month. This finding was similar to Groth (2005) who found that patients who were seen three or more times a week have more occurrences of contractures than those who were seen once or twice a week. Although contractures of the PIPJ were reportedly fairly uncommon at discharge, they surfaced commonly after discharge, similar to that reported by Groth (2005).

Customized orthoses were widely used with the wrist positioned at 20-30 degrees of flexion, metacarpophalangeal joint (MCPJ) in $60-70$ degrees in flexion, as typically recommended in the literature (Tang 2007, Groth 2005). Therapists at regional hospitals discontinue splinting at six weeks, and district hospitals at eight weeks. This is in line with international practices that support a five week or more splinting time to avoid the risk of tendon rupture. Groth (2005) also highlighted that discontinuation of splinting varies significantly as reported in the literature.

Timing of initiation of active range of movement was significantly different $(p=0.002)$ amongst different hospital settings. This may be attributed to the fact that patients present at different hospital settings at different points in their trajectory of post-operative recovery, thereby affecting the initiation of active movement. Related to this, tendon surgeries are performed at regional hospitals and patients are then referred to district hospitals for further rehabilitation. Groth (2005) noted that the cur- rent practice patterns for the initiation of active movement vary significantly. She stated that active flexion began at a mean of 18.6 postoperative days and the literature typically recommends 28 days. The findings of our study showed that about a quarter of therapists reported initiating exercises in the first, third and the fourth weeks post-operatively.

This study found that therapists began resistance exercises at five weeks. This finding was in line with what was reported by Rosenthal and Stoddard (2005) that resistance exercises need to be implemented later at five to eight weeks once the tendon is sufficiently healed.

As years of hand rehabilitation experience increased, respondents were more likely to see a tendon rupture indicating some level of inevitability to experiencing this complication (Groth 2005). Groth (2005) believes that sometimes specialization in hand rehabilitation cannot necessarily guarantee good flexor tendon outcomes.

The majority of therapists in the current study measured functional independence (70.8\%) and ROM (62\%). Groth (2005) also reported that therapists use outcome measures such as ROM, grip and pinch strength, Strickland's formula and function. Oltman et al (2008) state that functional outcome alone does not represent the true impact of flexor tendon injuries on patients.

Almost half (49\%) of the therapists are sometimes apprehensive about how to progress patients through rehabilitation. This finding is similar to that reported by Braga-Silva and Kuyven (2005). 


\section{CONCLUSION}

Therapists in this study managed patients depending on the demands in the setting in which they found themselves. The availability of protocols, and support by surgeons guided their decisions and choices. Rural therapy is symptomatic while that in urban and higher level hospitals is protocol driven. This study has shown the therapists' perspectives with regard to the management of repaired flexor tendons. The views of patients, orthopaedic surgeons and actual outcome data will add value to this study. It is recommended that minimal standards of care through the use of selected protocols be implemented in rural health care facilities.

\section{REFERENCES}

Braga-Silva J, Kuyven CRM 2005 Early active mobilization after flexor tendon repair in zone two. Chirugie de Main 24(3-4):165-168

Edinburg M, Widgerow AD, Biddulph S 1987 Early postoperative mobilization of the flexor tendon injuries using a modification of Kleinert technique. Journal of Hand Surgery 12A: 34-38

Groth GN 2008 Clinical decision making and therapist's autonomy in the context of Flexor Tendon Rehabilitation. Journal of Hand Therapy 21(3): 254259

Groth GN 2005 Current practice patterns of flexor tendon rehabilitation. Journal of Hand Therapy 18: 169-174

Jones NW, Laing H 2006 Acute injuries to the flexor and extensor tendons of the hand. Surgery International. 75:441-5

Lilly SI, Messer TM 2006 Complications after treatment of flexor tendon injuries. Journal of the American Academy of Orthopedic Surgery 14(7):387-396

Mackin EJ, Callahan AD, Skirven TM Schneider LH, Osterman AL. 2002 ed. Rehabilitation of The Hand and Upper Extremity (5th ed), ppl. 194-213, Mosby, St Louis

Mars M 2011 Telerehabilitation In South Africa Is There A Way Forward? International Journal of Telerehabilitation 3(1): 11-18

Oltman R, Neises G, Scheible D, Mehrtens D, Grunerberg C 2008 ICF components of corresponding outcome measures in flexor tendon rehabilitation-a systematic review. BMC Musculoskeletal Disorders 9(7):139

Rosenthal A, Stoddard CW 2005 Questions hand therapists ask about treatment of hand tendon injuries. Journal of Hand Therapy 18(2): 313-8

Sueoka SS, LaStayo PC 2008 Zone II flexor tendon rehabilitation: A proposed algorithm. Journal of Hand Therapy 21(4): 410- 13

Tang JB 2007 Indications, methods, post-operative motion and outcome evaluation of primary flexor tendon repairs in zone 2. Journal of Hand Surgery (Europe ) 32(2): $118-129$

Tidsskr NL 2008 Hand function after surgery for flexor tendon injuries. Journal of Hand Surgery 128 (1): $36-38$

Torie PAG, Atwal N, Sheriff D, Cowey A 2010 Flexor tendon injuries. Orthopaedics and Trauma 24(3): 217-222 